OPEN ACCESS

Edited by:

Wei Wei,

Anhui Medical University, China

Reviewed by:

Xin Hua Liu,

Anhui Medical University, China

Hong Wu,

Anhui University of Chinese Medicine,

China

${ }^{*}$ Correspondence:

Shuwen Liu

Liusw@smu.edu.cn

Kui Cheng

Chengk@smu.edu.cn

Specialty section:

This article was submitted to

Inflammation Pharmacology,

a section of the journal

Frontiers in Pharmacology

Received: 23 March 2018

Accepted: 19 July 2018

Published: 06 August 2018

Citation:

Cen X, Liu S and Cheng K (2018) The

Role of Toll-Like Receptor

in Inflammation and Tumor Immunity.

Front. Pharmacol. 9:878

doi: 10.3389/fphar.2018.00878

\section{The Role of Toll-Like Receptor in Inflammation and Tumor Immunity}

\author{
Xiaohong Cen, Shuwen Liu* and Kui Cheng* \\ Guangdong Provincial Key Laboratory of New Drug Screening, Guangzhou Key Laboratory of Drug Research for Emerging \\ Virus Prevention and Treatment, School of Pharmaceutical Sciences, Southern Medical University, Guangzhou, China
}

Toll-like receptors (TLRS) activation enables host to recognize a large number of pathogen-associated molecule patterns (PAMPs), ignite immune cells to discriminate between self and non-self, and then promote the following innate and adaptive immune responses. Accumulated clinical/preclinical evidences have proven TLRs to be critical role in the autoimmune diseases, including inflammatory and tumor-associated diseases. Activation of TLRs is becoming or has been a target for cancer treatment. It is shown that TLRs can induce preferable anti-tumor effect by eliciting inflammatory cytokines expression and cytotoxic T lymphocytes (CTLs) response. As adjuvant, TLRs agonists can launch a strong immune response to assist cancer radiotherapy and biochemotherapy. On the other hand, tumor-associated antigens acting as PAMPs, can also activate TLRs and induce tumor gene-related programmed cell death, including apoptosis, autophagy and programmed necrosis. While there are also arguments that the excessive TLRs expression will promote tumor deterioration in various organisms, as the TLR-induced inflammation will accelerate the cancer cells boost in the tumor microenvironment (TME). However, the effect of TLRs acting on cancers is still not quite clear today. In this review, we will summarize the recent researches of TLRs in cancer treatment and their role in TME, giving a brief overview on future expectation.

\footnotetext{
Keywords: Toll-like receptors, tumor microenvironment, programmed cell death, tumor immunotherapy, immune adjuvant
}

\section{INTRODUCTION}

As the best-characterized class of pattern recognized receptors (PRRs), most endosomal and plasma-membrane associated Toll-like receptors (TLRs) have been found to respond to conserved microbial products and endogenous damaged molecules such as lipopolysaccharide (LPS), lipopeptides, flagellin, bacterial DNA, viral double-stranded RNA (dsRNA), as well as high mobility group box 1 (HMGB1) and beta-defensins (O’Neill et al., 2009), which constitute the first-line of organismal defense against invading microbial pathogens, tissue injury or cancer, playing an important role in innate immune response and the subsequent of adaptive immune response (Kawai and Akira, 2011). TLRs have attracted a substantial of interest in cancer research due to the clinical relevance of TLRs expression and tumor control (Pradere et al., 2014). Accumulated evidences indicate that artificial activation of TLRs on the immune cells, including monocytes, macrophages, and dendritic cells (DCs), can generate strong efficacy to boost therapy-elicited anticancer immunity, characterized by priming CD8+ T-cell and natural killer cells (NK cells) (Mogensen, 2009; Cheadle et al., 2017). 
While TLRs are expressed not only in immune cells, but also tumor microenvironment (TME) (Gonzalez-Reyes et al., 2010). TME is a complex arrangement of cancer cells, normal cells, stromal tissue and extracellular cytokine. Stromal cells, the major components of TME, including cancer-associated fibroblasts (CAFs), tumor-associated macrophages (TAMs), marrow-derived suppressive cells (MDSCs), and regulatory $\mathrm{T}$ cells (Tregs) (Sato et al., 2009; Grivennikov et al., 2010). TLRs expressed among TME can not only induce self-programmed cell death, but also release cytokines and chemokine in the tumor environment, recruiting the immune cells to further release pro-inflammatory cytokines, pro-angiogenic factors and growth factors (Killeen et al., 2006; Zhou et al., 2009), such as TGF $\beta$, IL-8, CXCR4, ICAM-1, and VEGF, which may repair the anti-tumor function of antigen-presenting cells (APCs) and effector T-cells as well as apoptosis response. This inappropriate immune enhancement and anti-tumor immune through TLRs signaling pathway, will act as signal transducers to control tumor progression, metastasis, recurrence and chemotherapy tolerance (Connolly and O’Neill, 2012).

At present, tumor-antigen specific immune response and gene-regulated death are the two main strategies on cancer elimination by activate various signaling pathways and adaptor proteins. To better understand the complex interaction of antitumor effect through TLRs signaling pathway, we cover the recent advances of TLRs and cancer in this review, mainly according immunotherapy and cancer cells programmed death, hoping to make a contribution in novel strategies for variety of cancers.

\section{TLRs AND PROGRAMMED CELL DEATH}

\section{Programmed Cell Death}

Programmed cell death (PCD), systematically classified into apoptosis, autophagy and programmed necrosis, is proposed to be cell dying in a pathological state to keep host in equilibrium via specific cellular mechanism and various signaling pathways (Tan et al., 2009). Apoptosis, autophagy and programmed necrosis can be distinguished by their special morphological differences and physiological process (Zhivotovsky, 2011). All of them have certain association with each other and they may share the same signaling pathways or downstream effected adaptors to induce cancer cells death. It is suggested that apoptosis and programmed necrosis are the two manners of tumor cell death, while autophagy is contributed to both tumor cell death and survival (Ou et al., 2017). Molecules released from damaged issue, involving pathogens and cells toxic components are named damage-associated molecular patterns (DAMPs), which can be recognized by different TLRs to trigger various cellular response. Tumor-associated antigens released in the tumor environment, can act as DAMP to activate TLRs signaling pathway or downstream adaptor proteins, initiating different mechanism of PCD toward various cancer cells (Ouyang et al., 2012). Here, we give a brief of how TLRs are involved into the tumor cell death by different mechanism PCD and provide a guide for better tumor therapy. The intrinsic relation of TLRs and PCD toward tumor is showed in Figure 1.

\section{TLRs Induce Apoptosis in Tumor Cells}

Apoptosis, a gene controlled cell death to maintain internal environment homeostasis, is the most common type of PCD. Multiple evident have shown the correlation of TLRs and apoptosis that stimulation of hepatocellular carcinoma (HCC) with poly(I:C) (Table 1) can promote apoptosis by activation of TLR3 (Yoneda et al., 2008). Flagellin, a TLR5 agonist, was also found to induce HeLa cells death (Hancz et al., 2018). Mechanically, apoptosis conducted by TLRs is mainly divided into extrinsic and intrinsic way. The extrinsic pathway mediated the TNF receptor superfamily by TLR adaptor protein MYD88. It recruits the Fas-associated death domain (FADD) and pro-caspase- 8 to autocatalytic activation of caspase- 8 , leading the proteolysis of pro-caspase-3 into active caspase3 and triggering the apoptotic process (Meier and Vousden, 2007; Koizumi et al., 2016). On the other hand, under the control of mitochondrial pro-enzymes, the intrinsic pathway is to promote internal cytochrome $\mathrm{c}$ release into cytosol from the permeable mitochondrial membranes, and subsequently recruit Apaf-1 and pro-caspase-9, which will activate the downstream caspase-9/3 signaling cascade and then result in apoptosis (Ou et al., 2017). In addition, several studies have also demonstrated that TLRs are involved into different mechanism to induce apoptosis as well. For example, Imiquimod (IMQ) (Table 1), a synthetic nucleotide-like TLR 7 ligand, which has suggested to simultaneously induce ROS production to stimulate ATM/ATR pathway, leads to p53-dependent apoptosis in a skin basal cell carcinoma cell line (Huang et al., 2015). It is currently used as a topical and non-invasive treatment for superficial basal cell carcinoma (BCC), viral warts and other skin lesions in the clinic (Novak et al., 2008). In addition, TLRs can also disrupt the matrix metalloproteinase (MMP) to broke electron transfer chain and ATP synthesis, releasing the pro-apoptotic molecules to cytoplasm and consequently inducing tumor cells apoptosis (Reshma et al., 2016).

\section{TLRs Induce Autophagy in Tumor Cells}

Autophagy is characterized with the formation of doublemembrane-bound structure autophagosomes which warp the cancer cell or tumor-associated components with lysosomes and then degrade the contents in the capsule (Lin et al., 2013). Evident shows that autophagy are interconnected with apoptosis, as autophagy can either stimulate apoptosis by depleting tumorassociated suppressor, or impair tumor cells apoptosis by selectively reducing pro-apoptotic proteins, exhibiting dual effect on tumor control (Colell et al., 2007). The instinct of autophagy to remove the damaged proteins are crucial important to the last desperate efforts for survival of tumor cells. Various TLRs are proposed as autophagy inductor, which activate autophagy process by the downstream signaling adaptor MYD88 or TRIF, recruiting the TRAF6, and Beclin-1 to activate and generate autophagosomes (Shi and Kehrl, 2010). It is showed that IMQ (Table 1) can not only induce apoptosis in BCC, but also contribute to autophagic cell death in melanoma cell lines (Huang et al., 2015; Chang et al., 2017). However, Zhan et al. suggested that autophagy process induced by TLRs such as TLR3 and 


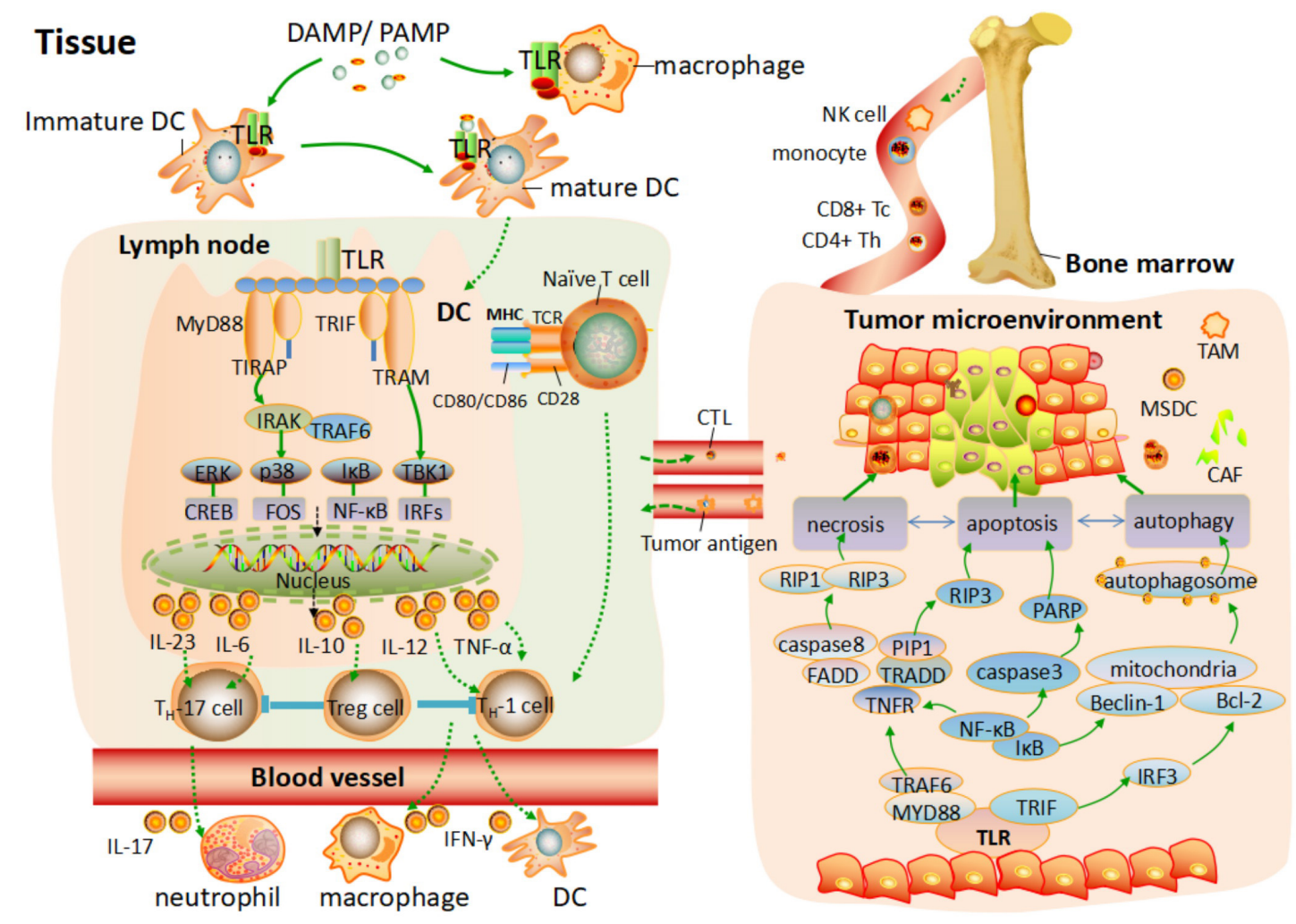

FIGURE 1 | TLRs mediated the T cells response and programmed cell death process toward tumor environment. After TLRs bind to PAMPs or DAMPs, the activation of pro-inflammatory response and different programmed cell death process are elicited. On one hand, various antigen-presenting cells (APC) are activated, and a programmed maturation is initiated to induce the migration of APC to lymph node. Different TLRs are activated by MYD88 and TRIF to elicit the downstream pathway, leading to the phosphorylation of NF-kB or IRFs and consequent transcription of various genes coding pro-inflammatory cytokine. This cytokine production response to PAMPs/DAMPs and activates different subset of T cells. In addition, naive T cells in the lymph node activate. Differentiated T cells migrate through the blood to the tissue or tumor environment and are further activated by different factor. On the other hand, tumor gene-related programmed cell death is elicited by activation of TLRs signaling pathway. Apoptosis, autophagy, and necrosis are independent but have certain association with each other, which share the same signaling pathways or downstream effected adaptor to induce cancer cell death.

TLR4, will also facilitate migration and invasion of lung cancer cells by release various harmful cytokines such as IL6, CCL2, and MMP2 (Zhan et al., 2014). In addition, the patients with higher TLR4 expression are showed more LC3II (a maker of autophagosomes) in their cancer associated fibroblasts (CAFs), which is in proportion to more aggressive relapse rate and poorer prognosis in 180 luminal breast patients (Zhao et al., 2017). To be noted that, for the dual effect of autophagy, the cancer treatment with TLRs agonists on autophagy cell death need a integrate consideration in clinical studies.

\section{TLRs Induce Programmed Necrosis in Tumor Cells}

Unlike apoptosis in a caspase-dependent manner, programmed necrosis is another mechanistically distinct cell death by phosphorylation and formation of RIP3 and PIR1 in a caspase-independent manner. In brief, necrosis, characterized by tremendous inflammatory response in cancer cells, enable to lyse mitochondrial and disrupt lysosomal membrane, leading to cell lysis or organelle dysfunction, which is crucial to maintain host in regular function (Cho et al., 2009). The study of the mechanism underlying TLR-mediated programmed necrosis is not quite clear. Nevertheless, TLR3 are proposed to induce necrosis partially dependent on TNF downstream signaling pathway and the subsequently to activate RIP adaptor protein to generate of RIP1 and RIP3 (Bianchi et al., 2017). It is also suggested that mouse colon carcinoma CT26 cells treated with poly(I:C) (Table 1) can not only induce an immune response, but also induce necrosis toward tumor cells exhibiting increased RIP1 and RIP3 interacting protein by TLR3/TICAM1-reactive oxygen species (Takaki et al., 2017). It shows that the underlying reaction between necrosis and TLRs are just beginning, more profound mystery is remain to explore.

\section{TLRs AND IMMUNE SYSTEM}

\section{TLRs Promote the Activation of Cytokines and Antitumor CTL Response}

Toll-like receptors play an important role in the innate immunity for the ability to recognize various molecular products derived 
TABLE 1 | Ongoing clinical study of TLRs agonists in cancer treatment as adjuvant.

\begin{tabular}{|c|c|c|c|c|c|}
\hline Compound & Target & Combination & Indication & Clinical phage & Reference \\
\hline \multirow[t]{5}{*}{ Poly ICLC } & TLR3 & Radiotherapy & Cutaneous T-cell lymphoma & Phase I & NCT02061449 \\
\hline & & NY-ESO-1 protein & Melanoma & Phase I/II & NCT01079741 \\
\hline & & autologous tumor lysate vaccine & Anaplastic astrocytoma & Phase I & NCT01204684 \\
\hline & & rhuFlt3L/CDX-301 & Low-grade B-cell lymphoma & Phase I/II & NCT01976585 \\
\hline & & Autologous OC-L Vaccine & Ovarian cancer & Phase I & NCT02452775 \\
\hline \multirow[t]{2}{*}{ GLA-SE } & TLR4 & Radiotherapy & Stage III/ IV Adult soft tissue sarcoma & Phase I & NCT02180698 \\
\hline & & MART-1 Antigen & II-IV Melanoma & Phase I & NCT02320305 \\
\hline G100 & TLR4 & Pembrolizumab & Follicular non-hodgkin's lymphoma & Phase I/II & NCT02501473 \\
\hline \multirow[t]{2}{*}{ Imiquimod } & TLR7 & Radiotherapy & Stage III or stage IV melanoma & Phase I & NCT00453050 \\
\hline & & Cyclophosphamide & Breast cancer & Phase I/II & NCT01421017 \\
\hline \multirow[t]{3}{*}{ Resiquimod (R848) } & TLR7/8 & NY-ESO-1 protein & Melanoma & Phase I & NCT00821652 \\
\hline & & MAGE-3 & Melanoma & Phase I & NCT00960752 \\
\hline & & Peptide vaccine (LPV7) & Melanoma & Phase I/II & NCT02126579 \\
\hline MEDI9197 & TLR $7 / 8$ & durvalumab & Solid tumors & Phase I & NCT02556463 \\
\hline \multirow[t]{4}{*}{ VTX-2337 } & TLR8 & Pegylated liposomal doxorubicin (PLD) & Ovarian cancer & Phase I & NCT01666444 \\
\hline & & Cyclophosphamide & Solid tumors & Phase I & NCT02650635 \\
\hline & & Cetuximab & Squamous cell cancer of head and neck & Phase I & NCT01334177 \\
\hline & & Radiotherapy & Low-grade B-cell lymphoma & Phase I/II & NCT01289210 \\
\hline MGN1703 & TLR9 & Ipilimumab & Advanced solid malignancies & Phase I & NCT02668770 \\
\hline CPG 7909 & TLR9 & Radiotherapy & Non-hodgkin lymphoma & Phase I & NCT00453050 \\
\hline \multirow[t]{5}{*}{ SD-101 } & TLR9 & Ipilimumab & Low-grade B-cell lymphoma & Phasel/II & NCT02254772 \\
\hline & & Pembrolizumab & Prostatic neoplasms & Phase I & NCT03007732 \\
\hline & & Ibrutinib & Grade 1-3A follicular lymphoma & Phasel/II & NCT02927964 \\
\hline & & Anti-OX40 antibody BMS & Low-grade B-cell lymphoma & Phase I & NCT03410901 \\
\hline & & Radiotherapy & Low-grade B-cell lymphoma & Phase I/II & NCT02254772 \\
\hline EMD 1201081 & TLR9 & 5-FU + Cisplatin + cetuximab & Squamous cell cancer of head and neck & Phase I & NCT01360827 \\
\hline
\end{tabular}

Data derived from http://clinicaltrials.gov.

from pathogens and endogenous molecules releases from cancer or dying cells (Vigneron, 2015), and subsequently boost immune response. In brief, when various TLRs on the immune cells bind to specific ligands, APCs such as macrophages and DCs are activated (Pulendran et al., 1999). Then, a programmed of maturation is initiated to induce the migration of APCs into lymph node and the accumulated cytokines such as TNF $\alpha$, IL6, IL12, MHC, and IFNs are subsequently released, which is crucial to the activation of NK cells and various cytotoxic T lymphocytes (CTLs) (Kopp and Medzhitov, 2003; Obermajer et al., 2018). For example, the lack of IFN- $\alpha$ in the tumor environment, deficits the ability of DC toward nucleic acids of tumor, leading to the immune tolerance and aggravation of cancer (Intidhar et al., 2012). While CpG ODN, an oligonucleotides agonists to TLR9, is reported to mediate TRIF downstream pathway to activate the IRF3 and IRF7, and then subsequently induce IFN- $\alpha$ and IFN- $\beta$ (Combes et al., 2017).

However, generation of $\mathrm{T}$ cells is an extremely complex process. CD80 and CD86, including in the B7 superfamily, are vital in activating $\mathrm{T}$ cells to produce cytokines and generate CTLs, while CD40 is essential in the priming phase to stimulate DC, which enable DCs migrate to secondary lymphoid organs (Sharpe and Freeman, 2002). T cell activation occurs only after the interaction of $\mathrm{T}$ cell receptor (TCR) with tumor-associated antigen-MHC complexes in the addition of CD28 costimulation with CD80 and CD86 (Sharma and Allison, 2015). Up regulation of adhesive and Co-stimulatory molecules on APC is required. Studies showed that activation of TLR4 can initiate the downstream pathway to increase the expression of CD80, CD86, CD40, and IL-12, which can promote the process of $\mathrm{T}$ cells (Clarke, 2000). Of note that, IL-12 can also play an important role in eliciting antigenpresenting process in CTLs, which skew the effector from an established Th2 to a Th1 response in immune cells (Heufler et al., 1996).

On the other hand, although CTLs can recognize and kill tumor cells to maintain the equilibrium of internal environment, playing a significant role in the tumor immunosurveillance, the $\mathrm{CD} 4+\mathrm{CD} 25+$ Foxp3 + regulatory Tregs induced by various mechanism, enable to counteract the antitumor CTL response, resulting in the poor immunogens and immunosurveillance escape of tumor cells (Sakaguchi, 2005; Vignali et al., 2008). It seems to be a good strategy to exert antitumor effect by down-regulation of Tregs. Studies showed that BLP, a synthetic bacterial lipoprotein, can depress Foxp3 expression to abrogate Treg function via TLR1/2 signaling pathway, and thus upregulate of CTLs (Zhang et al., 2011). In addition, DCs can also directly block the suppressive effect of $\mathrm{CD} 4+, \mathrm{CD} 25+$, and Treg cells, depending in part on IL-6 via TLRs signaling 
pathway (Pasare and Medzhitov, 2003). Of note, the process of $\mathrm{T}$ cell response mediated TLRs signaling pathway is showed in Figure 1.

\section{TLRs Agonists Act as Immune Adjuvant in the Cancer Therapy}

Approaches that aiming at co-activated pathways are widely developed. Treatment upon some cancer by combination of TLRs agonists with both radiotherapy and bio-chemotherapy, are suggested to exhibit positive immunologic effect and reduce immune tolerance. Evident also showed that TLR-based adjuvant has generated preferable efficacy and clinical responses for the cancer patients in some extend (He et al., 2015; Mahoney et al., 2015).

Radiotherapy is still the main non-surgical treatments for most cancer patients in the worldwide. It induced a lethal DNA damage, leading to the cellular death and mitotic catastrophe blocking (Delaney et al., 2005). Nevertheless, radiotherapy is insufficient to elicit adequate immune response to control the tumor in many of cases, other measures must be involved in (Formenti and Demaria, 2013; Schölch et al., 2015). TLRs agonists are proposed to be preferable adjuvant in many researches. It has demonstrated that systemically administrated TLR 4 agonists, potentiates the effect of radiotherapy in murine solid tumor with greater frequency of CTLs (Apetoh et al., 2007). Sandra et al. have also suggested that TLR7 agonist IMQ (Table 1) acting as adjuvant when combined with radiotherapy, can increase cytokine IL-10 expression and elicit T cell response, exhibiting good prognosis among breast cancer patients in the phase I/II clinical trial (Demaria et al., 2013). In addition, damage of radiation exposure from cancer radiotherapy is unavoidable (Singh et al., 2015). Of note, activation of TLRs can also contribute to hematopoietic replenishment as its upregulation of growth factor G-CSF, alleviating the intrinsic repair to radiant injuries (Singh et al., 2012; Kurkjian et al., 2017).

On the other hand, TLRs agonists can resist the tolerance in some of the cancers with combination of bio-chemotherapy. For example, Bacillus Calmette-Guerin (BCG), a ligand of TLR2 and TLR4, was used in the treatment of high-risk non-muscleinvasive bladder cancer against mycobacterium tuberculosis. However, antimicrobial peptides (AMPs) and massive proinflammatory cytokines were releases as the activation of TLRs via nuclear factor- $\kappa \mathrm{B}(\mathrm{NF}-\kappa \mathrm{B})$ and mitogen activated protein kinases (MAPK) pathway, resulting in the resistance toward mycobacteria (Akira et al., 2001; Ryffel et al., 2005). Evident showed that Poly (I:C) (Table 1) can induce the secretion of MHC class I molecules via TLR3 signaling pathway, increasing the tumor immunogenicity to overcome this resistance (Ayari et al., 2016). In addition, TLRs agonists can also elevate the efficacy in some of vaccine design. NY-ESO-1 is considered as a safe and compatible tumor antigen in design of vaccine, due to its extensive presence in various tumor types and the strong spontaneous cellular immune responses including activation of $\mathrm{CD} 4$ and $\mathrm{CD} 8+\mathrm{T}$ cells in the vitro and vivo study (Ayari et al., 2016). However, it is a pity that faint tumor regression is observed on cancer patients in the clinic research. Nina et al. has found that NY-ESO-1 in combination with a TLR7/8 agonist resiquimod (Table 1), can develop notable anti-NY-ESO-1 IgG antibody titers and enhanced $\mathrm{T}$ cells response in all high-risk melanoma patients, showing a predominant adjuvant effect of resiquimod in cancer treatment (Adams et al., 2008; Lubong et al., 2015). Altogether, these data show that activation of TLRs play an important role in the immune response, exhibiting particular prospect of TLRs agonists in the various cancer immunotherapy by acting as an adjuvant. It is worth to further investigate the adjuvant effect of TLRs agonists and achieve preferable vaccine application on the therapy of cancers and other diseases. The recently ongoing clinical studies of TLRs agonists in cancer treatment combined with other therapies are showed in Table 1.

\section{NEGATIVE EFFECT OF TLRs IN TUMOR PROGRESSION}

Although TLRs have generated significant efficacy in the cancer therapeutic studies and even in the clinical phages (Galluzzi et al., 2012), various scientists still query the effect as the existing studies show tumor progression, metastasis and recurrence in cancer therapy with the activation of TLRs (Grimmig et al., 2016; Ntoufa et al., 2016; Zhang et al., 2017). Tumor environment is a complex constitution of immune suppressive cells (Sato et al., 2009). However, TLRs can express not only in immune cells but also in this kind of tumorassociated cells and lead to tumor exacerbation. For example, the recurrent esophageal squamous cell carcinoma (EC) tissue exhibited significant higher expression of TLR3 and TLR4, which is associated with poor prognosis and a high probability of lymph node metastasis (Sheyhidin et al., 2011). In addition, cancer cells enable activate personal TLRs signals to release cytokines and chemokines in the tumor environment, which in turn to bind the immune suppressive cells and further release aberrant cytokines and chemokines, ultimately leading to tumor progression. Cancer cells from 133 prostate cancer patients have showed higher TLR3 and TLR9 expression accompanied with aberrant level of cytokines, inhibiting cell cycle induced apoptosis (González-Reyes et al., 2011). On the other hand, Liu et al. have also demonstrated that the tumor-derived snRNA-rich exosomes, can move to lung epithelial cells and activate the TLR3 to elicit multiple inflammatory cytokines release, resulting in the recruitment of neutrophils in second site to create the adaptive environment for primary tumor to metastasis (Liu et al., 2016). It shows that complicated interaction between tumor cells and immune cells in the tumor environment contributes to the aberrant immune enhancement and promote anti-tumor effect in TLRs signaling pathway. This can neatly illustrate the poor efficacy of some TLR agonists in the tumor clinical treatment. At present, the dual effects of TLRs regulators on tumors block the clinical practice. More and more studied are under intensively conducted for a systematical theory. Some of effective developed options are mentioned to solve this problem, such as targeted drug use 
and combination therapy, showing great advantages in clinical medication.

\section{CONCLUSION}

As the significant PRR, TLRs can defense host from invading pathogen, cancer cells and aberrant product by eliciting innate and adaptive immune response. In this review, we have highlighted the activation of TLRs in different phenotypes of cells that have the potential to stimulate immune response and $\mathrm{PCD}$ process to cancer cells. Although evident shows the contribution of TLRs to cancer treatment has generate a great effect, the pursuit to achieve an excellent treatment toward various tumors is still rough as the complexities and uncertainty of tumor environment. It remains effort to explore a bright and effective modality of tumor therapy in the future, and a better integration of different strategy upon cancer to achieve the maximum therapeutic efficacy. A fully exploit of the TLRs mechanism and

\section{REFERENCES}

Adams, S., O’neill, D. W., Nonaka, D., Hardin, E., Chiriboga, L., Siu, K., et al. (2008). Immunization of malignant melanoma patients with full-length NYESO-1 protein using toll-like receptor 7 agonist imiquimod as vaccine adjuvant. J. Immunol. 181, 776-784. doi: 10.4049/jimmunol.181.1.776

Akira, S., Takeda, K., and Kaisho, T. (2001). Toll-like receptors : critical proteins linking innate and acquired immunity. Nat. Immunol. 2, 675-680. doi: 10.1038/ 90609

Apetoh, L., Ghiringhelli, F., Tesniere, A., Obeid, M., Ortiz, C., Criollo, A., et al. (2007). Toll-like receptor 4-dependent contribution of the immune system to anticancer chemotherapy and radiotherapy. Nat. Med. 13, 1050-1059. doi: $10.1038 / \mathrm{nm} 1622$

Ayari, C., Besancon, M., Bergeron, A., Larue, H., Bussieres, V., and Fradet, Y. (2016). Poly(I:C) potentiates Bacillus Calmette-Guerin immunotherapy for bladder cancer. Cancer Immunol. Immunother. 65, 223-234. doi: 10.1007/ s00262-015-1789-y

Bianchi, F., Pretto, S., Tagliabue, E., Balsari, A., and Sfondrini, L. (2017). Exploiting poly(I:C) to induce cancer cell apoptosis. Cancer Biol. Ther. 18, 747-756. doi: 10.1080/15384047.2017.1373220

Chang, S. H., Huang, S. W., Wang, S. T., Chung, K. C., Hsieh, C. W., Kao, J. K., et al. (2017). Imiquimod-induced autophagy is regulated by ER stress-mediated PKR activation in cancer cells. J. Dermatol. Sci. 87, 138-148. doi: 10.1016/j.jdermsci. 2017.04.011

Cheadle, E. J., Lipowska-Bhalla, G., Dovedi, S. J., Fagnano, E., Klein, C., Honeychurch, J., et al. (2017). A TLR7 agonist enhances the antitumor efficacy of obinutuzumab in murine lymphoma models via NK cells and CD4 T cells. Leukemia 31, 1611-1621. doi: 10.1038/leu.2016.352

Cho, Y. S., Challa, S., Moquin, D., Genga, R., Ray, T. D., Guildford, M., et al. (2009). Phosphorylation-driven assembly of the RIP1-RIP3 complex regulates programmed necrosis and virus-induced inflammation. Cell 137, 1112-1123. doi: 10.1016/j.cell.2009.05.037

Clarke, S. (2000). The critical role of CD40/CD40L in the CD4-dependent generation of CD8+ T cell immunity. J. Leukoc. Biol. 67, 607-614. doi: 10.1002/ jlb.67.5.607

Colell, A., Ricci, J. E., Tait, S., Milasta, S., Maurer, U., Bouchier-Hayes, L., et al. (2007). GAPDH and autophagy preserve survival after apoptotic cytochrome c release in the absence of caspase activation. Cell 129, 983-997. doi: 10.1016/j. cell.2007.03.045

Combes, A., Camosseto, V., N'guessan, P., Argüello, R. J., Mussard, J., Caux, C., et al. (2017). BAD-LAMP controls TLR9 trafficking and signalling in human plasmacytoid dendritic cells. Nat. Commun. 8:913. doi: 10.1038/s41467-01700695- 1 modulators against tumor are desired and contributes to a better treatment of high-efficient, low-toxic, and well-tolerant to most cancer patients.

\section{AUTHOR CONTRIBUTIONS}

$\mathrm{XC}$ drafted the manuscript. KC and SL designed the project and revised the manuscript. All authors read and approved the final manuscript.

\section{FUNDING}

We thank the financial supports from National Natural Science Foundation of China (Nos. 81773558 and 81728007), start-up support in Southern Medical University of China (No. C1033269), and Youth Pearl River Scholar Program of Guangdong Province (No. C1034007) for this work.

Connolly, D. J., and O’Neill, L. A. (2012). New developments in Toll-like receptor targeted therapeutics. Curr. Opin. Pharmacol. 12, 510-518. doi: 10.1016/j.coph. 2012.06.002

Delaney, G., Jacob, S., Featherstone, C., and Barton, M. (2005). The role of radiotherapy in cancer treatment: estimating optimal utilization from a review of evidence-based clinical guidelines. Cancer 104, 1129-1137. doi: 10.1002/cncr. 21324

Demaria, S., Vanpouille-Box, C., Formenti, S. C., and Adams, S. (2013). The TLR7 agonist imiquimod as an adjuvant for radiotherapy-elicited in situ vaccination against breast cancer. Oncoimmunology 2:e25997. doi: 10.4161/onci. 25997

Formenti, S. C., and Demaria, S. (2013). Combining radiotherapy and cancer immunotherapy: a paradigm shift. J. Natl. Cancer Inst. 105, 256-265. doi: 10.1093/jnci/djs629

Galluzzi, L., Vacchelli, E., Eggermont, A., Fridman, W. H., Galon, J., SautesFridman, C., et al. (2012). Trial watch: experimental toll-like receptor agonists for cancer therapy. Oncoimmunology 1, 699-716. doi: 10.4161/onci.20696

González-Reyes, S., Fernández, J. M., González, L. O., Aguirre, A., Suárez, A., González, J. M., et al. (2011). Study of TLR3, TLR4, and TLR9 in prostate carcinomas and their association with biochemical recurrence. Cancer Immunol. Immunother. 60, 217. doi: 10.1007/s00262-010-0931-0

Gonzalez-Reyes, S., Marin, L., Gonzalez, L., Gonzalez, L. O., Del Casar, J. M., Lamelas, M. L., et al. (2010). Study of TLR3, TLR4 and TLR9 in breast carcinomas and their association with metastasis. BMC Cancer 10:665. doi: 10.1186/1471-2407-10-665

Grimmig, T., Moench, R., Kreckel, J., Haack, S., Rueckert, F., Rehder, R., et al. (2016). Toll like receptor 2, 4, and 9 signaling promotes autoregulative tumor cell growth and VEGF/PDGF expression in human pancreatic cancer. Int. J. Mol. Sci. 17:E2060. doi: 10.3390/ijms17122060

Grivennikov, S. I., Greten, F. R., and Karin, M. (2010). Immunity, inflammation, and cancer. Cell 140, 883-899. doi: 10.1016/j.cell.2010. 01.025

Hancz, D., Szabo, A., Molnar, T., Varga, Z., Hancz, A., Gregus, A., et al. (2018). Flagellin increases death receptor-mediated cell death in a RIP1-dependent manner. Immunol. Lett. 193, 42-50. doi: 10.1016/j.imlet.2017.11.007

He, L. Z., Weidlick, J., Sisson, C., Marsh, H. C., and Keler, T. (2015). Tolllike receptor agonists shape the immune responses to a mannose receptortargeted cancer vaccine. Cell. Mol. Immunol. 12, 719-728. doi: 10.1038/cmi.20 14.100

Heufler, C., Koch, F., Stanzl, U., Topar, G., Wysocka, M., Trinchieri, G., et al. (1996). Interleukin-12 is produced by dendritic cells and mediates T helper 1 development as well as interferon-gamma production by T helper 1 cells. Eur. J. Immunol. 26, 659-668. doi: 10.1002/eji.1830260323 
Huang, S. W., Chang, S. H., Mu, S. W., Jiang, H. Y., Wang, S. T., Kao, J. K., et al. (2015). Imiquimod activates p53-dependent apoptosis in a human basal cell carcinoma cell line. J. Dermatol. Sci. 81, 182-191. doi: 10.1016/j.jdermsci.2015. 12.011

Intidhar, L. G. S., Isabelle, T., Sophie, G. L., Jean-Damien, C., Jean-Yves, B., Isabelle, R. C., et al. (2012). Plasmacytoid dendritic cells infiltrating ovarian cancer are associated with poor prognosis. Oncoimmunology 1, 380-382. doi: 10.4161/ onci.18801

Kawai, T., and Akira, S. (2011). Toll-like receptors and their crosstalk with other innate receptors in infection and immunity. Immunity 34, 637-650. doi: 10.1016/j.immuni.2011.05.006

Killeen, S. D., Wang, J. H., Andrews, E. J., and Redmond, H. P. (2006). Exploitation of the toll-like receptor system in cancer: a doubled-edged sword? $\mathrm{Br}$. J. Cancer 95, 247-252. doi: 10.1038/sj.bjc.6603275

Koizumi, Y., Nagase, H., Nakajima, T., Kawamura, M., and Ohta, K. (2016). Toll-like receptor 3 ligand specifically induced bronchial epithelial cell death in caspase dependent manner and functionally upregulated fas expression. Allergol. Int. 65(Suppl.), S30-S37. doi: 10.1016/j.alit.2016.05.006

Kopp, E., and Medzhitov, R. (2003). Recognition of microbial infection by tolllike receptors. Curr. Opin. Immunol. 15, 396-401. doi: 10.1016/S0952-7915(03) 00080-3

Kurkjian, C. J., Guo, H., Montgomery, N. D., Cheng, N., Yuan, H., Merrill, J. R., et al. (2017). The toll-like Receptor $2 / 6$ agonist, FSL-1 lipopeptide, therapeutically mitigates acute radiation syndrome. Sci. Rep. 7:17355. doi: 10.1038/s41598-017-17729-9

Lin, C., Tsai, S. C., Tseng, M. T., Peng, S. F., Kuo, S. C., Lin, M. W., et al. (2013). AKT serine/threonine protein kinase modulates baicalin-triggered autophagy in human bladder cancer T24 cells. Int. J. Oncol. 42, 993-1000. doi: 10.3892/ijo. 2013.1791

Liu, Y., Gu, Y., Han, Y., Zhang, Q., Jiang, Z., Zhang, X., et al. (2016). Tumor exosomal RNAs promote lung pre-metastatic niche formation by activating alveolar epithelial TLR3 to recruit neutrophils. Cancer Cell 30, 243-256. doi: 10.1016/j.ccell.2016.06.021

Lubong, S. R., Pavlick, A., Gnjatic, S., Cruz, C. M., Vengco, I., Hasan, F., et al. (2015). Resiquimod as an immunologic adjuvant for NY-ESO-1 protein vaccination in patients with high risk melanoma. Cancer Immunol. Res. 3, 278-287. doi: 10.1158/2326-6066.CIR-14-0202

Mahoney, K. M., Rennert, P. D., and Freeman, G. J. (2015). Combination cancer immunotherapy and new immunomodulatory targets. Nat. Rev. Drug Discov. 14, 561-584. doi: 10.1038/nrd4591

Meier, P., and Vousden, K. H. (2007). Lucifer's labyrinth-ten years of path finding in cell death. Mol. Cell 28, 746-754. doi:10.1016/j.molcel.2007. 11.016

Mogensen, T. H. (2009). Pathogen recognition and inflammatory signaling in innate immune defenses. Clin. Microbiol. Rev. 22, 240-273. doi: 10.1128/CMR. 00046-08

Novak, N., Yu, C. F., Bieber, T., and Allam, J. P. (2008). Toll-like receptor 7 agonists and skin. Drug News Perspect. 21, 158-165.

Ntoufa, S., Vilia, M. G., Stamatopoulos, K., Ghia, P., and Muzio, M. (2016). Toll-like receptors signaling: a complex network for NF-kappaB activation in B-cell lymphoid malignancies. Semin. Cancer Biol. 39, 15-25. doi: 10.1016/j. semcancer.2016.07.001

Obermajer, N., Urban, J., Wieckowski, E., Muthuswamy, R., Ravindranathan, R., Bartlett, D. L., et al. (2018). Promoting the accumulation of tumor-specific T cells in tumor tissues by dendritic cell vaccines and chemokine-modulating agents. Nat. Protoc. 13, 335-357. doi: 10.1038/nprot.2017.130

O’Neill, L. A., Bryant, C. E., and Doyle, S. L. (2009). Therapeutic targeting of Tolllike receptors for infectious and inflammatory diseases and cancer. Pharmacol. Rev. 61, 177-197. doi: 10.1124/pr.109.001073

Ou, L., Lin, S., Song, B., Liu, J., Lai, R., and Shao, L. (2017). The mechanisms of graphene-based materials-induced programmed cell death: a review of apoptosis, autophagy, and programmed necrosis. Int. J. Nanomedicine 12, 6633-6646. doi: 10.2147/IJN.S140526

Ouyang, L., Shi, Z., Zhao, S., Wang, F. T., Zhou, T. T., Liu, B., et al. (2012). Programmed cell death pathways in cancer: a review of apoptosis, autophagy and programmed necrosis. Cell Prolif. 45, 487-498. doi: 10.1111/j.1365-2184. 2012.00845.x
Pasare, C., and Medzhitov, R. (2003). Toll pathway-dependent blockade of CD4+ CD25+ T cell-mediated suppression by dendritic cells. Science 299, 1033-1036. doi: $10.1126 /$ science.1078231

Pradere, J. P., Dapito, D. H., and Schwabe, R. F. (2014). The yin and yang of toll-like receptors in cancer. Oncogene 33, 3485-3495. doi: 10.1038/onc.2013.302

Pulendran, B., Smith, J. L., Caspary, G., Brasel, K., Pettit, D., Maraskovsky, E., et al. (1999). Distinct dendritic cell subsets differentially regulate the class of immune response in vivo. Proc. Natl. Acad. Sci. U.S.A. 96, 1036-1041. doi: 10.1073/pnas.96.3.1036

Reshma, S. C., Syama, S., and Mohanan, P. V. (2016). Nano-biointeractions of PEGylated and bare reduced graphene oxide on lung alveolar epithelial cells: a comparative in vitro study. Colloids Surf. B Biointerfaces 140, 104-116. doi: 10.1016/j.colsurfb.2015.12.030

Ryffel, B., Fremond, C., Jacobs, M., Parida, S., Botha, T., Schnyder, B., et al. (2005). Innate immunity to mycobacterial infection in mice: critical role for toll-like receptors. Tuberculosis 85, 395-405. doi: 10.1016/j.tube.2005.08.021

Sakaguchi, S. (2005). Naturally arising Foxp3-expressing CD25+CD4+ regulatory $\mathrm{T}$ cells in immunological tolerance to self and non-self. Nat. Immunol. 6, 345-352. doi: 10.1038/ni1178

Sato, Y., Goto, Y., Narita, N., and Hoon, D. S. (2009). Cancer cells expressing tolllike receptors and the tumor microenvironment. Cancer Microenviron. 2(Suppl. 1), 205-214. doi: 10.1007/s12307-009-0022-y

Schölch, S., Rauber, C., Tietz, A., Rahbari, N. N., Bork, U., Schmidt, T., et al. (2015). Radiotherapy combined with TLR7/8 activation induces strong immune responses against gastrointestinal tumors. Oncotarget 6, 4663-4676. doi: 10.18632/oncotarget.3081

Sharma, P., and Allison, J. P. (2015). The future of immune checkpoint therapy. Science 348, 56-61. doi: 10.1126/science.aaa 8172

Sharpe, A. H., and Freeman, G. J. (2002). The B7-CD28 superfamily. Nat. Rev. Immunol. 2, 116-126. doi: 10.1038/nri727

Sheyhidin, I., Nabi, G., Hasim, A., Zhang, R. P., Ainiwaer, J., Ma, H., et al. (2011). Overexpression of TLR3, TLR4, TLR7 and TLR9 in esophageal squamous cell carcinoma. World J. Gastroenterol. 17, 3745-3751. doi: 10.3748/wjg.v17.i32. 3745

Shi, C. S., and Kehrl, J. H. (2010). TRAF6 and A20 regulate lysine 63-linked ubiquitination of Beclin-1 to control TLR4-induced autophagy. Sci. Signal. 3:ra42. doi: 10.1126/scisignal.2000751

Singh, V. K., Ducey, E. J., Fatanmi, O. O., Singh, P. K., Brown, D. S., Purmal, A. et al. (2012). CBLB613: a TLR 2/6 Agonist, Natural lipopeptide of Mycoplasma arginini, as a novel radiation countermeasure. Radiat. Res. 177, 628-642. doi: $10.1667 / R R 2657.1$

Singh, V. K., Romaine, P. L., and Seed, T. M. (2015). Medical countermeasures for radiation exposure and related injuries: characterization of medicines, fdaapproval status and inclusion into the strategic national stockpile. Health Phys. 108, 607-630. doi: 10.1097/HP.0000000000000279

Takaki, H., Shime, H., Matsumoto, M., and Seya, T. (2017). Tumor cell death by pattern-sensing of exogenous RNA: tumor cell TLR3 directly induces necroptosis by poly(I:C) in vivo, independent of immune effector-mediated tumor shrinkage. Oncoimmunology 6:e1078968. doi: 10.1080/2162402X.2015. 1078968

Tan, M. L., Ooi, J. P., Ismail, N., Moad, A. I., and Muhammad, T. S. (2009). Programmed cell death pathways and current antitumor targets. Pharm. Res. 26, 1547-1560. doi: 10.1007/s11095-009-9895-1

Vignali, D. A., Collison, L. W., and Workman, C. J. (2008). How regulatory T cells work. Nat. Rev. Immunol. 8, 523-532. doi: 10.1038/nri2343

Vigneron, N. (2015). Human Tumor Antigens and Cancer Immunotherapy. Biomed. Res. Int. 2015:948501. doi: 10.1155/2015/948501

Yoneda, K., Sugimoto, K., Shiraki, K., Tanaka, J., Beppu, T., Fuke, H., et al. (2008). Dual topology of functional Toll-like receptor 3 expression in human hepatocellular carcinoma: differential signaling mechanisms of TLR3-induced NF-kappaB activation and apoptosis. Int. J. Oncol. 33, 929-936.

Zhan, Z., Xie, X., Cao, H., Zhou, X., Zhang, X. D., Fan, H., et al. (2014). Autophagy facilitates TLR4- and TLR3-triggered migration and invasion of lung cancer cells through the promotion of TRAF6 ubiquitination. Autophagy 10, 257-268. doi: 10.4161/auto. 27162

Zhang, D., Li, L., Jiang, H., Knolhoff, B. L., Lockhart, A. C., Wang-Gillam, A., et al. (2017). Constitutive IRAK4 activation underlies poor prognosis and 
chemoresistance in pancreatic ductal adenocarcinoma. Clin. Cancer Res. 23, 1748-1759. doi: 10.1158/1078-0432.CCR-16-1121

Zhang, Y., Luo, F., Cai, Y., Liu, N., Wang, L., Xu, D., et al. (2011). TLR1/TLR2 agonist induces tumor regression by reciprocal modulation of effector and regulatory T cells. J. Immunol. 186, 1963-1969. doi: 10.4049/jimmunol.1002320

Zhao, X. L., Lin, Y., Jiang, J., Tang, Z., Yang, S., Lu, L., et al. (2017). High-mobility group box 1 released by autophagic cancer-associated fibroblasts maintains the stemness of luminal breast cancer cells. J. Pathol. 243, 376-389. doi: 10.1002/ path.4958

Zhivotovsky, B. (2011). "Systems biology analysis of cell death pathways in cancer: how collaborative and interdisciplinary research helps," in Cancer Systems Biology, Bioinformatics and Medicine, eds A. Cesario and F. Marcus (Dordrecht: Springer).

Zhou, M., Mcfarland-Mancini, M. M., Funk, H. M., Husseinzadeh, N., Mounajjed, T., and Drew, A. F. (2009). Toll-like receptor expression in normal ovary and ovarian tumors. Cancer Immunol. Immunother. 58, 1375-1385. doi: 10.1007/s00262-008-0650-y

Conflict of Interest Statement: The authors declare that the research was conducted in the absence of any commercial or financial relationships that could be construed as a potential conflict of interest.

The reviewer XL and handling editor declared their shared affiliation.

Copyright (c) 2018 Cen, Liu and Cheng. This is an open-access article distributed under the terms of the Creative Commons Attribution License (CC BY). The use, distribution or reproduction in other forums is permitted, provided the original author(s) and the copyright owner(s) are credited and that the original publication in this journal is cited, in accordance with accepted academic practice. No use, distribution or reproduction is permitted which does not comply with these terms. 